\title{
FATTY ACIDS COMPOSITION IN FRUITS OF WILD ROSE SPECIES
}

\author{
RENATA NOWAK \\ Chair and Department of Pharmaceutical Botany \\ Skubiszewski Medical University of Lublin \\ Chodźki 1, 20-093 Lublin, Poland \\ e-mail: renata.nowak@am.lublin.pl
}

(Received: January 11, 2005. Accepted: March 10, 2005)

\begin{abstract}
The oil content and fatty acids profile of a number of Polish wild species of rose fruits were examined by GC. The total fatty acid contents ranged from $6.5 \%$ to $12.9 \%$ of dry mass in fruits. The composition of oils was similar in the investigated species.

17 components were identified. An average composition was estimated as follows: linoleic acid (44.4-55.7\%), $\alpha$-linolenic acid (18.6-31.4\%), oleic acid (13.5-20.3\%), palmitic acid (2.3-3.3\%), stearic acid (1-2.5\%), octadecenoic acid $(0.38-0.72 \%)$, eicosenoic acid $(0.3-0.7 \%)$, eicosadienoic acid $(0-0.16 \%)$, erucic acid $(0.03-0.17 \%)$ and minor fatty acids.

The results indicate that rose fruits are a rich source of unsaturated fatty acids, especially in $R$. rubiginosa, $R$. rugosa and $R$. dumalis.

There were statistically significant $(\mathrm{p}<0.05)$ differences in fatty acid compositions of some species. Fatty acids were suggested to have a potential chemotaxonomic value in this genus.
\end{abstract}

KEY WORDS: Fatty acids composition, Rosa L. fruits, GC analysis.

\section{INTRODUCTION}

The genus Rosa L. (Rosaceae) comprises about 150 wild species distributed in Europe, Asia, the Middle East and North America. Twenty five species (fourteen native) of the genus, mostly belonging to Caninae Section, are known in the flora of Poland area (Zieliński 1987).

Systematic relationships within the genus Rosa are confusing due to the variability of species and the weak barriers to intraspecific hybridization. Usually, the primary descriptors used by taxonomists concerned with the genus Rosa, are morphological features.

Many criteria have been used to support some taxonomic proposals such as seed morphology (Buth and Misri 1984), flower colour (Yokoi 1975), pollen exine (Ueda and Okada 1994), chromosome number (Lata 1982), DNA amount and polymorphism (Rajapakse et al. 1992; Matsumoto et al. 1997, 1998) and isozymes (Grossi et al. 1997, 1998). Some chemical markers, mainly phenolic compounds, have been used, too (Biolley et al. 1994; Mikanagi et al. 1995; Raymond et al. 1995).

Chemotaxonomic significance of fatty acids in other plants has been previously reported (Valesco and Goffman 1999; Sanina et al. 2004). There is no data concerning the possible role of these group of compounds in $R o-$ $s a$ L. genus.

Rose hips have been used both in treatment and in food industry for many years for their rich chemical composition
(Hodisan et al. 1997; Hornero-Méndez and Míngues-Mosquera 2000; Razungles et al. 1989; Hvattum 2002; Nowak and Krzaczek 1994), and interesting pharmacological properties (Daels-Rakotoarison 2002; Karakaya and Kavas 1999; Kumarasamy et al. 2002; Winther et al. 1999; Umezu et al. 2002). Fruits of Rosa canina L. and the pure active substance - galactolipide (GOPO) isolated from fruits showed anti-inflammatory properties (Larsen et al. 2003; Rein et al. 2004).

Rose fruits - nuts, usually represent a waste material during production of pharmaceutical and nourishing medicaments, juices, syrups, jams, tea and alcoholic beverages after fermentation. In the meantime they are mainly an underestimated source of valuable oil containing unsaturated fatty acids in cosmetic industry. They are a rich source of proteins, starch, vitamin E, sterols, minerals and carotenoids, too (Stepanov et al. 1983; Zlatanov 1989; Cisowski et al. 1995; Ozcan 2002; Szentmihalyi et al. 2002).

The data mentioned in references show that content of oils and fatty acids in some of rose species, especially $R$. canina L., $R$. rubiginosa L. and $R$. rugosa Thunb., were partly investigated, however there is lack of complex comparing examinations of other species in this field (Malec et al. 1993; Cisowski et al. 1995; Ozcan 2002).

In the study comparison of the amount and composition of fatty acids, especially unsaturated ones, from some rose species growing commonly in Lubelszczyzna region of Poland, was established to evaluate their pharmaceutical properties. In addition the taxonomic aspects were discussed, too. 


\section{MATERIAL AND METHODS}

\section{Plant material}

Rose hips were collected from bushes widely growing in Kazimierz Dolny near Lublin in Poland in September 2002 and authenticated by Prof. Dr T. Krzaczek. Names of spe- cies are those by Zieliński $(1985,1987)$ and varieties by Popek (1996). A voucher specimens have been deposited in the herbarium of Department of Pharmaceutical Botany, Medical University, in Lublin. The examined species are given in Table 1.

TABLE 1. Plant material.

\begin{tabular}{|c|c|c|c|c|}
\hline Taxa no. & Sections, species, varieties & Synonym names & Place of collection & Data of collection \\
\hline & & Sectio Caninae DC. Em. Christ. & & \\
\hline \multirow{3}{*}{1} & $R$. rubiginosa $\mathrm{L} .=R$. eglanteria $\mathrm{L}$. & & & \\
\hline & R. rubiginosa var. rubiginosa & $\begin{array}{l}\text { R. comosa Ripart in Schultz } \\
\text { R. rubiginosa var. typica Heinr. Braun in Beck }\end{array}$ & $\begin{array}{l}\text { Męćmierz } \\
\text { Bochotnica }\end{array}$ & $\begin{array}{l}02-09-19 \\
02-09-19\end{array}$ \\
\hline & R. dumalis Bechst. & & & \\
\hline 2 & R. vosagiaca Desportes & R.dumalis var. afzeliana (Fr.) Boulenger & $\begin{array}{l}\text { Kazimierz D. } \\
\text { Bochotnica } \\
\text { Bochotnica }\end{array}$ & $\begin{array}{l}02-09-19 \\
02-09-19 \\
02-09-19\end{array}$ \\
\hline 3 & R. subcanina (Christ) Dalla Torre et Sarnath. & & $\begin{array}{l}\text { Kazimierz D. } \\
\text { Bochotnica } \\
\text { Męćmierz }\end{array}$ & $\begin{array}{l}02-09-19 \\
02-09-19 \\
02-09-23\end{array}$ \\
\hline 4 & R. dumalis Bechst. var. bsseriana Popek & R. caryophyllaceae Besser pro parte & $\begin{array}{l}\text { Kazimierz } \\
\text { Panasówka } \\
\text { Męćmierz }\end{array}$ & $\begin{array}{l}02-09-19 \\
02-09-23 \\
02-09-23\end{array}$ \\
\hline 5 & R. coriifolia Fries & R.dumalis var. coriifolia $(\mathrm{Fr}$.) Boulenger & $\begin{array}{l}\text { Kazimierz D. } \\
\text { Bochotnica } \\
\text { Męćmierz } \\
\text { Panasówka }\end{array}$ & $\begin{array}{l}02-09-19 \\
02-09-19 \\
02-09-23 \\
02-09-23\end{array}$ \\
\hline
\end{tabular}

R. canina $\mathrm{L}$.

R. canina L. var. canina R. canina L. var. typica Braun

R. canina L. var. typica Braun

Kazimierz D.

02-09-19

Kazimierz

02-09-19

Mẹćmierz

02-09-19

Kazimierz D.

02-09-19

Bochotnica

02-09-23

Męćmierz

02-09-23

Bohotnica

02-09-19

8

R. canina L. var dumalis Baker

R. canina var. transitoria $\mathrm{R}$. Keller

Józefów

02-09-29

Kazimierz D.

02-09-05

\section{R. inodora Fries}

R. inodora Fries var. inodora

R. agrestis Savi var. inodora (Fries) Borbás

Kazimierz D.

Kazimierz D.

Męćmierz

Panasówka

\section{R. villosa $\mathbf{L}$.}

(Bess.) Chrshan.

\section{Sectio Cinnamomea DC.}

\section{R. rugosa Thunb.}

$\begin{array}{ll}\text { Olsztyn } & 02-09-15 \\ \text { Lublin } & 02-09-28 \\ \text { Kazimierz D. } & 02-09-05\end{array}$




\section{Fatty acids extraction and methylation}

The standard procedure used for analyzing the fatty acid contents of plant material was as follows.

The dried and powdered (FP IV1965; FP VI 2002) fruits - nuts $(20 \mathrm{~g})$ were extracted with cold n-hexane in Soxhlet apparatus and the solvent was evaporated off under reduced pressure.

Oil content was determined by Soxhlet extraction, using n-hexane and METHOD 1.122. described by IUPAC (1979). The obtained oil samples were methylated directly with $14 \% \mathrm{BF} 3-\mathrm{MeOH}$ and fatty acid methyl esters were analyzed using GLC (Stołyhwo et al. 1987).

\section{Gas chromatography}

A Hewlett-Packard Model 6890 chromatograph equipped with flame ionization detector FID was used. The column was $25 \mathrm{~m}$ long, $0.25 \mathrm{~mm}$ I.D., packed with fused silica.

The injection port and detector were at $235^{\circ} \mathrm{C}$ with helium flow rate of $55 \mathrm{ml} / \mathrm{min}$. Optimum temperature programme was: $175-210^{\circ} \mathrm{C}$ at $1.5^{\circ} \mathrm{C} / \mathrm{min}$.

Results were quantified by measurement of individual peak area to total peaks area of fatty acids with the aid of the HP 3396 Integrator.

The identification of the compounds was performed by comparison of their retention time with data of the authentic samples.
All solvents used were of chromatographic grade (Merck, Germany). Fatty acid methyl ester standards were obtained from Sigma (Germany).

\section{Statistical analysis}

Samples were studied in triplicate, analyses were carried out in parallel, and then the averages were calculated. The statistical analysis of MANOVA, Levene's tests and Tukey HSD test for homogeneity of variances were performed to evaluate the significance of differences between values at the level of $\mathrm{p}<0.05$ and taxonomic distance of investigated rose species.

\section{RESULTS AND DISCUSSION}

The content of oil in rose fruits of particular species ranged from $6.5 \%$ to $12.9 \%$ (Fig. 1). The highest amount of oil ( $>10 \%)$ was stated in the fruits of $R$. canina var. dumalis, $R$. dumalis var. besseriana and $R$. subcanina. All of the investigated oils showed a high quantity of essential unsaturated fatty acids ranging from $70.9 \%$ to $79.56 \%$, which was considered promising for pharmaceutical and nutrient purposes (Fig. 2).

GC analyses show the fatty acid distribution of the eleven investigated rose taxa (Table 2).
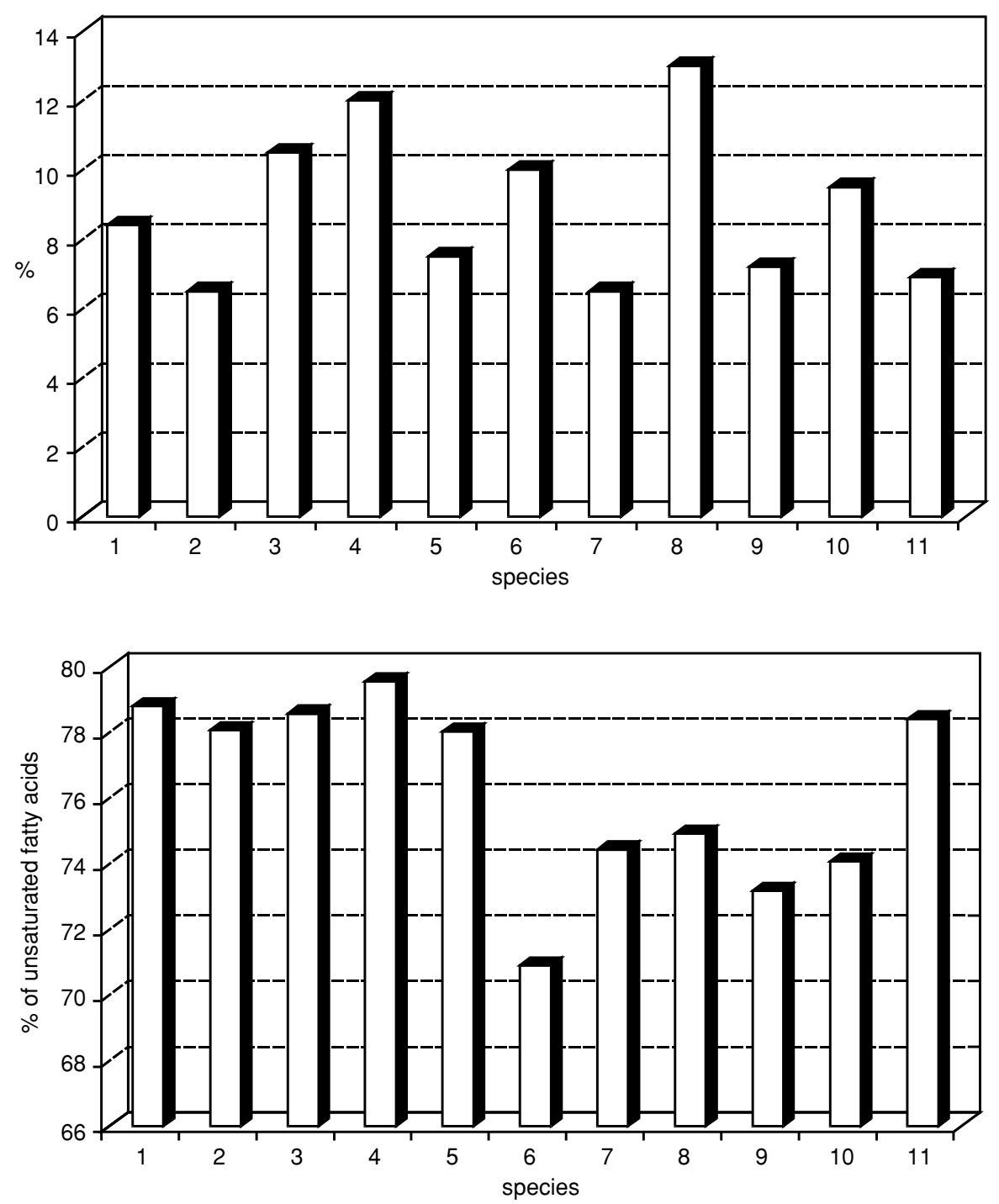

Fig. 1. The content of oil in rose fruits (explanation: the number of species are given in Table 1).
Fig. 2. Quantity of essential unsaturated fatty acids (\%) in the investigated fruit of wild rose species (the number of species are given in Table 1). 
TABLE 2. Fatty acids composition in fruits of wild rose species ( $\%$ means $\pm S D, n=3)$.

\begin{tabular}{|c|c|c|c|c|c|c|c|c|c|c|c|}
\hline \multirow[b]{3}{*}{ Fatty acid } & \multicolumn{11}{|c|}{ Species } \\
\hline & 1 & 2 & 3 & 4 & 5 & 6 & 7 & 8 & 9 & 10 & 11 \\
\hline & $\begin{array}{c}R . \\
\text { rubiginosa }\end{array}$ & $\begin{array}{c}\text { R. dumalis } \\
\text { var. } \\
\text { dumalis }\end{array}$ & $\begin{array}{c}R . \\
\text { subcanina }\end{array}$ & $\begin{array}{l}\text { R. dumalis } \\
\text { var. } \\
\text { besseriana } \\
\text { Popek }\end{array}$ & $\begin{array}{l}\text { R. dumalis } \\
\text { var. } \\
\text { coriifolia }\end{array}$ & $\begin{array}{l}\text { R. canina } \\
\text { var. } \\
\text { canina }\end{array}$ & $\begin{array}{l}\text { R. canina } \\
\text { var. } \\
\text { corym- } \\
\text { bifera }\end{array}$ & $\begin{array}{l}R \text {. canina } \\
\text { var. } \\
\text { dumalis }\end{array}$ & $\begin{array}{c}\text { R. inodora } \\
\text { var. } \\
\text { inodora }\end{array}$ & $\begin{array}{l}\text { R. villosa } \\
\text { subsp. } \\
\text { mollis }\end{array}$ & R. rugosa \\
\hline 1. $\mathrm{C}_{14: 0}$ & $\begin{array}{l}0.052 \\
\pm 0.01\end{array}$ & $\begin{array}{l}0.035 \\
\pm 0.01\end{array}$ & $\begin{array}{c}0.04 \\
\pm 0.01\end{array}$ & $\begin{array}{c}0.03 \\
\pm 0.01\end{array}$ & $\begin{array}{l}0.035 \\
\pm 0.01\end{array}$ & $\begin{array}{c}0.03 \\
\pm 0.01\end{array}$ & $\begin{array}{c}0.05 \\
\pm 0.01\end{array}$ & $\begin{array}{c}0.04 \\
\pm 0.01\end{array}$ & $\begin{array}{c}0.05 \\
\pm 0.01\end{array}$ & $\begin{array}{c}0.04 \\
\pm 0.01\end{array}$ & $\begin{array}{c}0.03 \\
\pm 0.01\end{array}$ \\
\hline 2. $\mathrm{C}_{15: 0}$ & $\begin{array}{l}0.035 \\
\pm 0.01\end{array}$ & $\begin{array}{l}0.028 \\
\pm 0.01\end{array}$ & $\begin{array}{c}0.03 \\
\pm 0.01\end{array}$ & $\begin{array}{l}0.035 \\
\pm 0.01\end{array}$ & $\begin{array}{c}0.03 \\
\pm 0.01\end{array}$ & $\begin{array}{c}0.03 \\
\pm 0.01\end{array}$ & $\begin{array}{c}0.03 \\
\pm 0.01\end{array}$ & $\begin{array}{c}0.04 \\
\pm 0.01\end{array}$ & $\begin{array}{c}0.04 \\
\pm 0.01\end{array}$ & $\begin{array}{c}0.04 \\
\pm 0.05\end{array}$ & $\begin{array}{c}0.04 \\
\pm 0.01\end{array}$ \\
\hline 3. $\mathrm{C}_{16: 0}$ & $\begin{array}{c}2.86 \\
\pm 0.12\end{array}$ & $\begin{array}{c}2.93 \\
\pm 0.10\end{array}$ & $\begin{array}{c}3.09 \\
\pm 0.07\end{array}$ & $\begin{array}{c}2.79 \\
\pm 0.22\end{array}$ & $\begin{array}{c}2.34 \\
\pm 0.09\end{array}$ & $\begin{array}{c}3.54 \\
\pm 0.03\end{array}$ & $\begin{array}{c}3.23 \\
\pm 0.17\end{array}$ & $\begin{array}{c}3.10 \\
\pm 0.05\end{array}$ & $\begin{array}{c}3.24 \\
\pm 0.04\end{array}$ & $\begin{array}{c}3.07 \\
\pm 0.19\end{array}$ & $\begin{array}{c}2.77 \\
\pm 0.08\end{array}$ \\
\hline 4. $C_{16: 1, n-9}$ & $\begin{array}{l}0.035 \\
\pm 0.01\end{array}$ & $\begin{array}{l}0.052 \\
\pm 0.01\end{array}$ & $\begin{array}{c}0.03 \\
\pm 0.01\end{array}$ & $\begin{array}{l}0.035 \\
\pm 0.01\end{array}$ & $\begin{array}{l}0.036 \\
\pm 0.01\end{array}$ & $\begin{array}{c}0.05 \\
\pm 0.01\end{array}$ & $\begin{array}{c}0.04 \\
\pm 0.01\end{array}$ & $\begin{array}{c}0.04 \\
\pm 0.01\end{array}$ & $\begin{array}{c}0.05 \\
\pm 0.01\end{array}$ & $\begin{array}{c}0.07 \\
\pm 0.02\end{array}$ & $\begin{array}{c}0.04 \\
\pm 0.01\end{array}$ \\
\hline 5. $\quad \mathrm{C}_{16: 1, \mathrm{n}-7}$ & $\begin{array}{c}0.02 \\
\pm 0.01\end{array}$ & $\begin{array}{c}0.09 \\
\pm 0.01\end{array}$ & $\begin{array}{c}0.07 \\
\pm 0.01\end{array}$ & $\begin{array}{l}0.069 \\
\pm 0.01\end{array}$ & $\begin{array}{c}0.05 \\
\pm 0.01\end{array}$ & $\begin{array}{c}0.06 \\
\pm 0.01\end{array}$ & $\begin{array}{c}0.08 \\
\pm 0.01\end{array}$ & $\begin{array}{c}0.08 \\
\pm 0.01\end{array}$ & $\begin{array}{c}0.09 \\
\pm 0.01\end{array}$ & $\begin{array}{c}0.08 \\
\pm 0.01\end{array}$ & $\begin{array}{c}0.18 \\
\pm 0.02\end{array}$ \\
\hline 6. $\mathrm{C}_{17: 0}$ & $\begin{array}{c}0.05 \\
\pm 0.01\end{array}$ & $\begin{array}{l}0.063 \\
\pm 0.01\end{array}$ & $\begin{array}{c}0.05 \\
\pm 0.01\end{array}$ & $\begin{array}{c}0.06 \\
\pm 0.01\end{array}$ & $\begin{array}{c}0.05 \\
\pm 0.01\end{array}$ & $\begin{array}{c}0.08 \\
\pm 0.02\end{array}$ & $\begin{array}{c}0.06 \\
\pm 0.01\end{array}$ & $\begin{array}{c}0.04 \\
\pm 0.01\end{array}$ & $\begin{array}{c}0.05 \\
\pm 0.01\end{array}$ & $\begin{array}{c}0.07 \\
\pm 0.01\end{array}$ & $\begin{array}{c}0.05 \\
\pm 0.01\end{array}$ \\
\hline 7. $\mathrm{C}_{18: 0}$ & $\begin{array}{c}1.64 \\
\pm 0.03\end{array}$ & $\begin{array}{c}1.78 \\
\pm 0.05\end{array}$ & $\begin{array}{c}1.89 \\
\pm 0.09\end{array}$ & $\begin{array}{c}1.77 \\
\pm 0.02\end{array}$ & $\begin{array}{c}1.78 \\
\pm 0.02\end{array}$ & $\begin{array}{c}2.46 \\
\pm 0.11\end{array}$ & $\begin{array}{c}2.06 \\
\pm 0.07\end{array}$ & $\begin{array}{c}2.05 \\
\pm 0.04\end{array}$ & $\begin{array}{c}2.40 \\
\pm 0.09\end{array}$ & $\begin{array}{l}1.73 \\
\pm 0.1\end{array}$ & $\begin{array}{c}1.04 \\
\pm 0.05\end{array}$ \\
\hline 8. $\quad \mathrm{C}_{18: 1, \Delta 6}$ & $\begin{array}{c}0.03 \\
\pm 0.01\end{array}$ & $\begin{array}{c}0.07 \\
\pm 0.01\end{array}$ & $\begin{array}{l}0.065 \\
\pm 0.01\end{array}$ & $\begin{array}{l}0.077 \\
\pm 0.02\end{array}$ & $\begin{array}{c}0.07 \\
\pm 0.01\end{array}$ & $\begin{array}{c}0.02 \\
\pm 0.01\end{array}$ & $\begin{array}{c}0.07 \\
\pm 0.01\end{array}$ & $\begin{array}{c}0.06 \\
\pm 0.01\end{array}$ & $\begin{array}{c}0.05 \\
\pm 0.01\end{array}$ & $\begin{array}{c}0.08 \\
\pm 0.02\end{array}$ & $\begin{array}{c}0.05 \\
\pm 0.01\end{array}$ \\
\hline 9. $\quad \mathrm{C}_{18: 1, \Delta 9}$ & $\begin{array}{l}14.22 \\
\pm 0.86\end{array}$ & $\begin{array}{l}14.40 \\
\pm 0.49\end{array}$ & $\begin{array}{r}13.88 \\
\pm 0.38\end{array}$ & $\begin{array}{l}13.54 \\
\pm 0.21\end{array}$ & $\begin{array}{l}15.26 \\
\pm 0.71\end{array}$ & $\begin{array}{l}20.30 \\
\pm 1.09\end{array}$ & $\begin{array}{c}17.6 \\
\pm 0.54\end{array}$ & $\begin{array}{l}17.17 \\
\pm 0.12\end{array}$ & $\begin{array}{l}18.22 \\
\pm 0.33\end{array}$ & $\begin{array}{l}18.41 \\
\pm 0.46\end{array}$ & $\begin{array}{r}14.42 \\
\pm 0.54\end{array}$ \\
\hline 10. $\mathrm{C}_{18: 1, \Delta 11}$ & $\begin{array}{c}0.57 \\
\pm 0.06\end{array}$ & $\begin{array}{c}0.52 \\
\pm 0.07\end{array}$ & $\begin{array}{c}0.49 \\
\pm 0.03\end{array}$ & $\begin{array}{c}0.43 \\
\pm 0.07\end{array}$ & $\begin{array}{c}0.38 \\
\pm 0.04\end{array}$ & $\begin{array}{c}0.46 \\
\pm 0.05\end{array}$ & $\begin{array}{c}0.46 \\
\pm 0.03\end{array}$ & $\begin{array}{c}0.55 \\
\pm 0.02\end{array}$ & $\begin{array}{c}0.47 \\
\pm 0.03\end{array}$ & $\begin{array}{c}0.52 \\
\pm 0.05\end{array}$ & $\begin{array}{c}0.72 \\
\pm 0.09\end{array}$ \\
\hline 11. $\mathrm{C}_{18: 2, \Delta 9,12}$ & $\begin{array}{l}47.20 \\
\pm 0.24\end{array}$ & $\begin{array}{l}48.42 \\
\pm 0.07\end{array}$ & $\begin{array}{l}49.77 \\
\pm 0.96\end{array}$ & $\begin{array}{l}53.77 \\
\pm 0.92\end{array}$ & $\begin{array}{l}55.51 \\
\pm 0.87\end{array}$ & $\begin{array}{l}51.67 \\
\pm 1.80\end{array}$ & $\begin{array}{l}55.70 \\
\pm 1.21\end{array}$ & $\begin{array}{l}54.55 \\
\pm 0.17\end{array}$ & $\begin{array}{l}50.29 \\
\pm 0.68\end{array}$ & $\begin{array}{l}44.41 \\
\pm 0.37\end{array}$ & $\begin{array}{l}50.32 \\
\pm 0.94\end{array}$ \\
\hline 12. $\mathrm{C}_{18: 3, \Delta 9,12,15}$ & $\begin{array}{l}31.40 \\
\pm 0.31\end{array}$ & $\begin{array}{l}29.52 \\
\pm 0.34\end{array}$ & $\begin{array}{l}28.69 \\
\pm 0.21\end{array}$ & $\begin{array}{l}25.61 \\
\pm 0.42\end{array}$ & $\begin{array}{l}22.34 \\
\pm 0.26\end{array}$ & $\begin{array}{r}19.08 \\
\pm 0.12\end{array}$ & $\begin{array}{l}18.60 \\
\pm 0.14\end{array}$ & $\begin{array}{l}20.24 \\
\pm 0.51\end{array}$ & $\begin{array}{l}22.79 \\
\pm 0.63\end{array}$ & $\begin{array}{l}29.49 \\
\pm 0.24\end{array}$ & $\begin{array}{l}27.90 \\
\pm 0.28\end{array}$ \\
\hline 13. $\mathrm{C}_{20: 0}$ & $\begin{array}{c}0.74 \\
\pm 0.04\end{array}$ & $\begin{array}{c}0.73 \\
\pm 0.03\end{array}$ & $\begin{array}{c}0.74 \\
\pm 0.01\end{array}$ & $\begin{array}{c}0.77 \\
\pm 0.04\end{array}$ & $\begin{array}{c}0.77 \\
\pm 0.02\end{array}$ & $\begin{array}{c}0.90 \\
\pm 0.04\end{array}$ & $\begin{array}{c}0.81 \\
\pm 0.03\end{array}$ & $\begin{array}{c}0.85 \\
\pm 0.03\end{array}$ & $\begin{array}{c}0.77 \\
\pm 0.07\end{array}$ & $\begin{array}{c}0.83 \\
\pm 0.05\end{array}$ & $\begin{array}{c}0.72 \\
\pm 0.02\end{array}$ \\
\hline 14. $\mathrm{C}_{20: 1}$ & $\begin{array}{c}0.36 \\
\pm 0.01\end{array}$ & $\begin{array}{c}0.41 \\
\pm 0.02\end{array}$ & $\begin{array}{c}0.44 \\
\pm 0.01\end{array}$ & $\begin{array}{c}0.32 \\
\pm 0.01\end{array}$ & $\begin{array}{c}0.38 \\
\pm 0.04\end{array}$ & $\begin{array}{c}0.30 \\
\pm 0.01\end{array}$ & $\begin{array}{c}0.31 \\
\pm 0.01\end{array}$ & $\begin{array}{c}0.42 \\
\pm 0.02\end{array}$ & $\begin{array}{c}0.30 \\
\pm 0.03\end{array}$ & $\begin{array}{c}0.59 \\
\pm 0.04\end{array}$ & $\begin{array}{c}0.70 \\
\pm 0.03\end{array}$ \\
\hline 15. $\mathrm{C}_{20: 2}$ & $\begin{array}{c}0.13 \\
\pm 0.01\end{array}$ & $\begin{array}{c}0.11 \\
\pm 0.01\end{array}$ & $\begin{array}{c}0.09 \\
\pm 0.02\end{array}$ & $\begin{array}{c}0.11 \\
\pm 0.01\end{array}$ & $\begin{array}{c}0.14 \\
\pm 0.01\end{array}$ & $\begin{array}{c}0.08 \\
\pm 0.01\end{array}$ & $\begin{array}{c}0.09 \\
\pm 0.01\end{array}$ & nd & $\begin{array}{c}0.07 \\
\pm 0.01\end{array}$ & nd & $\begin{array}{c}0.16 \\
\pm 0.02\end{array}$ \\
\hline 16. $\mathrm{C}_{22: 0}$ & $\begin{array}{c}0.15 \\
\pm 0.01\end{array}$ & $\begin{array}{c}0.13 \\
\pm 0.01\end{array}$ & $\begin{array}{c}0.21 \\
\pm 0.01\end{array}$ & $\begin{array}{c}0.20 \\
\pm 0.01\end{array}$ & $\begin{array}{c}0.15 \\
\pm 0.01\end{array}$ & $\begin{array}{c}0.15 \\
\pm 0.01\end{array}$ & $\begin{array}{c}0.13 \\
\pm 0.02\end{array}$ & nd & $\begin{array}{c}0.15 \\
\pm 0.01\end{array}$ & nd & $\begin{array}{c}0.29 \\
\pm 0.03\end{array}$ \\
\hline 17. $\mathrm{C}_{22: 1}$ & $\begin{array}{c}0.08 \\
\pm 0.01\end{array}$ & $\begin{array}{l}0.025 \\
\pm 0.01\end{array}$ & $\begin{array}{c}0.03 \\
\pm 0.01\end{array}$ & $\begin{array}{c}0.07 \\
\pm 0.02\end{array}$ & $\begin{array}{c}0.04 \\
\pm 0.01\end{array}$ & $\begin{array}{c}0.07 \\
\pm 0.01\end{array}$ & $\begin{array}{c}0.05 \\
\pm 0.01\end{array}$ & $\begin{array}{c}0.14 \\
\pm 0.03\end{array}$ & $\begin{array}{c}0.03 \\
\pm 0.01\end{array}$ & $\begin{array}{c}0.17 \\
\pm 0.02\end{array}$ & $\begin{array}{c}0.04 \\
\pm 0.01\end{array}$ \\
\hline
\end{tabular}

nd - not detected

17 components were identified. The major acids in rose oil of the examined species are linoleic and linolenic acids. An average composition was estimated as follows: linoleic acid (44.4-55.7\%), $\alpha$-linolenic acid (18.6-31.4\%), oleic acid (13.5-20.3\%), palmitic acid (2.3-3.3\%), stearic acid (1-2.5\%), octadecanoic acid (0.38-0.72\%), eicosenoic acid (0.3-0.7\%), eicosadienoic acid (0-0.16\%), erucic acid $(0.03-0.17 \%)$ and minor fatty acids.
The five major acids are palmitic, stearic, oleic, linoleic and linolenic. Collectively these five acids comprise about $97 \%$ of total fatty acids in all investigated roses.

The qualitative composition of oil was similar in the investigated species. However, some differences were observed.

In Table 3 the approximate relative ratios of some acids (C18:0/C16:0; C18:0/C16:1, n-7; C18:0/C18:1, $\Delta 9$; 
TABLE 3. Factors which might be useful in distinguishing fatty acids of Rosa species: C18:0/C16:0, C18:0/C16:1, ${ }_{\mathrm{n}-7} ; \mathrm{C} 18: 0 / \mathrm{C} 18: 1,{ }_{\Delta 9} ; \mathrm{C} 18: 0 / \mathrm{C} 18: 1,{ }_{\Delta 11}$; C18:0/C18:2, ${ }_{\Delta 9,12} ; \mathrm{C} 18: 0 / \mathrm{C} 18: 3,{ }_{\Delta 9,12,15} ; \mathrm{C} 18: 0 / \mathrm{C} 20: 0$ ratios.

\begin{tabular}{|c|c|c|c|c|c|c|c|c|c|c|c|}
\hline \multirow[b]{3}{*}{ Fatty acid } & \multicolumn{11}{|c|}{ Species } \\
\hline & 1 & 2 & 3 & 4 & 5 & 6 & 7 & 8 & 9 & 10 & 11 \\
\hline & $\begin{array}{c}R . \\
\text { rubiginosa }\end{array}$ & $\begin{array}{c}\text { R. dumalis } \\
\text { var. } \\
\text { dumalis }\end{array}$ & $\begin{array}{c}R . \\
\text { subcanina }\end{array}$ & $\begin{array}{l}\text { R. dumalis } \\
\text { var. } \\
\text { besseriana } \\
\text { Popek }\end{array}$ & $\begin{array}{l}\text { R. dumalis } \\
\text { var. } \\
\text { coriifolia }\end{array}$ & $\begin{array}{l}\text { R. canina } \\
\text { var. } \\
\text { canina }\end{array}$ & $\begin{array}{l}\text { R. canina } \\
\text { var. } \\
\text { corym- } \\
\text { bifera }\end{array}$ & $\begin{array}{c}\text { R. canina } \\
\text { var. } \\
\text { dumalis }\end{array}$ & $\begin{array}{c}\text { R. inodora } \\
\text { var. } \\
\text { inodora }\end{array}$ & $\begin{array}{l}\text { R. villosa } \\
\text { subsp. } \\
\text { mollis }\end{array}$ & R. rugosa \\
\hline 3. $\mathrm{C}_{16: 0}$ & 1.74 & 1.65 & 1.63 & 1.58 & 1.32 & 1.44 & 1.57 & 1.61 & 1.35 & 1.77 & 2.66 \\
\hline 5. $\quad \mathrm{C}_{16: 1, \mathrm{n}-7}$ & 82 & 19.8 & 27 & 25.3 & 35.6 & 41 & 25.8 & 25.6 & 26.7 & 21.6 & 5.8 \\
\hline 7. $\mathrm{C}_{18: 0}$ & 1 & 1 & 1 & 1 & 1 & 1 & 1 & 1 & 1 & 1 & 1 \\
\hline 9. $\quad \mathrm{C}_{18: 1, \Delta 9}$ & 8.67 & 8.08 & 7.34 & 7.65 & 8.57 & 8.25 & 8.54 & 8.38 & 7.59 & 10.64 & 13.87 \\
\hline 10. $\mathrm{C}_{18: 1, \Delta 11}$ & 2.88 & 3.42 & 3.86 & 4.12 & 4.68 & 5.35 & 4.48 & 3.73 & 5.11 & 3.33 & 1.44 \\
\hline 11. $\mathrm{C}_{18: 2, \Delta 9,12}$ & 28.8 & 27.2 & 26.33 & 30.38 & 31.19 & 21 & 27.04 & 26.61 & 20.95 & 25.67 & 48.34 \\
\hline 12. $\mathrm{C}_{18: 3, \Delta 9,12,15}$ & 19.1 & 16.58 & 15.18 & 14.47 & 12.55 & 7.75 & 9.03 & 9.87 & 9.50 & 17.05 & 26.83 \\
\hline 13. $\mathrm{C}_{20: 0}$ & 2.22 & 2.44 & 2.55 & 2.3 & 2.31 & 2.73 & 2.54 & 2.41 & 3.12 & 2.08 & 1.44 \\
\hline
\end{tabular}

C18:0/C18:1, $\Delta 11 ; \mathrm{C} 18: 0 / \mathrm{C} 18: 2, \Delta 9,12 ; \mathrm{C} 18: 0 / \mathrm{C} 18: 3 \Delta 9$, 12, 15; C18:0/C20:0) were reported. Chemotaxonomic significance of fatty acids in plants, e.g. in Boroginaceae, has been previously reported (Valesco and Goffman 1999; Sanina et al. 2004) and such factors as these have been used in chemotaxonomy in the past, too (Nagy and Nordby 1974).

It proved that the ratios in the four $R$. dumalis varieties (samples No 2-5) were very similar. The three $R$. canina varieties (No 6-8) were very similar, too. However, these two groups of species were noticeably different. Especially ratios $\mathrm{C} 18: 3, \Delta 9,12,15$ / C18:0 were always higher in species belonging to $R$. dumalis group. They ranged from 12.55 to 16.58 and from 7.75 to 9.87 for $R$. dumalis and $R$.

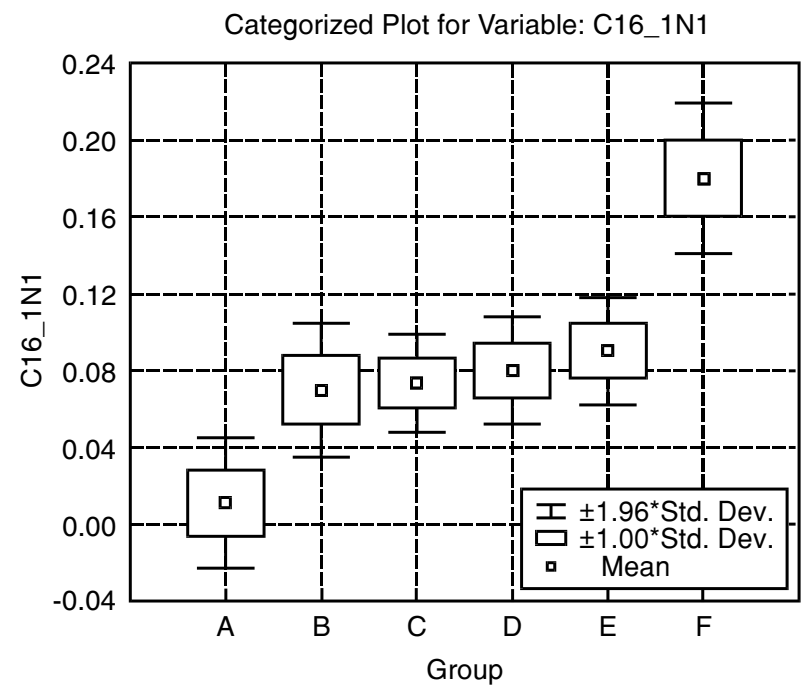

Fig. 3. Categorized diagram for variable C16:1, n-7 (Tukey HSD test). Groups: A: R. rubiginosa; B: $R$. dumalis var. dumalis, $R$. subcanina, $R$. dumalis. var. besseriana, $R$. dumalis var. coriifolia; C: $R$. canina var. canina, $R$. canina var. corymbifera, $R$. canina var. dumalis; D: $R$. inodora var. inodora; E: R. villosa L. subsp. mollis; F: R. rugosa. canina species groups respectively). The composition of major fatty acids in $R$. inodora is quite similar to $R$. canina species. $R$. rubiginosa and $R$. villosa possess similar relative content of major fatty acids (ratios) approximate to $R$. dumalis species group. However, R. rugosa always differs markedly from other species. This is caused by highest amount of the $\mathrm{C} 18$ unsaturated acids and smaller content of stearic acid, what is manifested in analyzed ratios. The analyzed ratios are significantly different for $R$. rugosa and similar for all other species.

The performed observations are in high agreement with systematic distance of the analyzed species. $R$. rugosa belongs to sectio Cinnamomea; however the other species are from sectio Caninae. The statistical Tukey HSD test, performed for some variables, showed a very high similarity between most rose species from Caninae sectio (except $R$. rubiginosa) and the large distance of $R$. rugosa, too (Fig. 3 ). This may suggest that these factors might be useful in chemotaxonomy of Rosa L. species. They can also be useful in determination of the species, which become the most valuable material from the medical point of view.

The biochemistry of lipid metabolism in oil fruits has been recently reviewed (Salas et al. 2000). The products of fatty acid syntheses are mainly $\mathrm{C} 16$ or C18 saturated acyl chains. However, most plants oils are rich in certain types of unsaturated fatty acids, such as oleate and linoleate. This is due to plants having the necessary mechanisms to introduce double bonds into specific positions of the acyl chains yielded by the fatty acid synthesis reactions. Lipid biosynthesis is also affected by environmental factors, e.g. light, temperature, water stress, soil and atmospheric constituents, pest attack (Salas et al. 2000). In this work, the plant material was collected in the same stage of development of fruits and from the same region with similar environmental factors to diminish their influence on fatty acid composition.

The shown data reveal that types of $R$. dumalis, R. rubiginosa and $R$. rugosa contain the highest concentration of 
unsaturated fatty acids. Therefore, they become a much more attractive source of these compounds in comparison with the other analyzed rose taxa.

Dietary unsaturated fatty acids are essential for correct functioning of human organism (Crawford et al. 2000; Kato 2000). Dietary intake of linolenic acid is for example associated with a decreased risk of cardiovascular-diseases mortality and is responsible for antidiabetic, antimicrobial and cardio-protective activities (Ziegler 1989; Gurr 1992; French et al. 1997; Frenoux et al. 2001; Djousse et al. 2003).

Rose oils are a rich source of polyunsaturated fatty acids, especially linoleic and linolenic acid. It is favorable for medicinal and nutritional application of these natural products.

\section{LITERATURE CITED}

BIOLLEY J.P., JAY M., VIRICEL M.R. 1994. Flavonoid diversity and metabolism in 100 Rosa x hybrida cultivars. Phytochemistry. 35: 413-419.

BUTH G.M., MISRI K. 1984. Seed structure of some species of Rosaceae. J. Pl. Anat. Morph. 1: 63-68.

CISOWSKI W., ZIELIŃSKA-STASIEK M., STOŁYHWO A. 1995. Research of raw plant material rich in oils EFAs and oleinic acid. Herba Polon. 41 (4): 165-169.

CRAWFORD M., GALLI C., VISIOLI F., RENAUD S., SIMOPOULUS A.P., SPECTOR A.A. 2000. Role of plant-derived omega-3 fatty acids in human nutrition. Annales of Nutr. Metab. 44: 263-265.

DAELS-RAKOTOARISON D.A., GRESSIER B., TROTIN F., BRUNET C., LUYCKX M., DINE T., BAILLEUL F., CAZIN M., CAZIN J.-C. 2002. Effects of Rosa canina fruit extract on neutrophil respiratory burst. Phytother. Res. 16: 157-161.

DJOUSSE L., HUNT S.C., ARNETT D.K., PROVINCE M.A., ECKFELDT J.H., ELLISON J. 2003. Dietary linolenic acid is inversely associated with plasma triacylglycerol. Am. J. Clin. Nutr. 78: 1098-1102.

Farmakopea Polska IV. 1965. PZWL, Warszawa. (in Polish)

Farmacopea Polska VI. 2002. PTF, Warszawa. (in Polish)

FRENCH M.A., PARROTT A.M., KIELO E.S., RAJOTTE R.V., WANG L.C., THOMSON A.B., CLANDINI M.T. 1997. Polyunsaturated fat in the diet may improve intestinal function in patients with Crohn's disease. Biochem. Biophys. Acta 1360: 262-270.

FRENOUX J.M.R., PROST E.D., BELLEVILLE J.L., PROST J.L. 2001. A polyunsaturated fatty acid diet lowers blood pressure and improves antioxidant status in spontaneously hypertensive rats. J. Nutr. 131: 39-45.

GURR M.I. 1992. Dietary lipids and coronary heart disease: Old evidence, new perspective. Progr. Lipid Res. 31 (3): 195-243.

GROSSI C., RAYMOND O., JAY M. 1997. Isozyme polymorphism of Rosa spp. And cultivar identification. Euphytica 98: 11-19.

GROSSI C., RAYMOND O., JAY M. 1998. Flavonoid and enzyme polymorphisms and taxonomic organization of Rosa sections: Carolinae, Cinnamomeae, Pimpinellifoliae and Synstyleae. Biochem. Syst. Ecol. 26: 857-871.

HODISAN T., SOCACIU C., ROPAU I., NEAMTU I. 1997. Carotenoid composition of Rosa canina fruits determined by thin - layer chromatography and high-performance liquid chromatography. J. Pharm. Biomed. Anal. 16: 521-528.

HORNERO-MÉNDEZ D., MÍNGUES-MOSQUERA M.I. 2000. Carotenoid pigments in Rosa mosqueta hips, an alternative carotenoid source for foods. J. Agric. Food Chem. 48: 825-828.

HVATTUM E. 2002. Determination of phenolic compounds in rose hip (Rosa canina) using liquid chromatography coupled to electrospray ionization tandem mass spectrometry and diode-array detection. Rapid Commun. Mass Spectr. 16: 665.

IUPAC (1979), Standard methods for the analysis of the oils, fats and derivatives ( $6^{\text {th }}$ ed.). Oxford: Pergamon Press.

KATO M., MIURA T., NAKAO M., IWAMOTO N., ASHIDA T., TANIGAWA L. 2000. Effect of alpha-linolenic acid on blood glucose, insulin and GLUT4 protein content of type 2 diabetic mice. J. Health Sci. 46: 489-492.

KARAKAYA S., KAVAS, A. 1999. Antimutagenic activities of some foods. J. Sci. Food Agric. 79: 237-241.

KUMARASAMY Y., COX P.J., JASPARS M., NAHAR L., SARKER S.D. 2002. Screening seeds of Scottish plants for antibacterial activity. J. Ethnopharm. 83: 73-77.

LATA P. 1982. Cytological studies in the genus Rosa. III. Meiotic analysis of ten cultivars. Caryologia. 47: 639-647.

LARSEN E., KHARAZMI A., CHRISTENSEN L.P., CHRISTENSEN S.B. 2003. An antiinflammatory galactolipid from rose-hip (Rosa canina) that inhibits chemotaxis of human peripheral blood neutrophils in vitro. J. Nat. Prod. 66: 994-995.

MALEC I.S., CIVEIRA M.E., VIGO M.S. 1993. Seeds of Rosa rubiginosa L. (Rosa mosqueta). Chemical composition of seed meal residue. Ann. Asoc. Quim. Arg. 81: 445-450.

MATSUMOTO S., WAKITA H., FUKAI H. 1997. Molecular classification of wild roses using organelle DNA probes. Sci. Hortic. 68: 191-196.

MATSUMOTO S., KOUCHI M., YABUKI J., KUSUNOKI M., UEDA Y., FUKUI H. 1998. Phylogenetic analyses of the genus Rosa using the matK sequence: molecular evidence for the narrow genetic background of modern roses. Sci. Hort. 77: $73-$ $-82$.

MIKANAGI Y., YOKOI M., UEDA Y., SAITO, N. 1995. Flower flavonol and anthocyanin distribution in subgenus Rosa. Biochem. Systems Ecol. 23: 183-200.

NAGY S., NORDBY H.E. 1974. Fatty acids of triglycerides from Citrus juice sacs. Phytochemistry 13: 153-157.

NOWAK R., KRZACZEK T. 1994. Flavonoids from Rosa pomifera var. ciliato-petala Bess. (Chrshan.). Acta Pol. Pharm. 51: 407-410.

OZCAN M. 2002. Nutrient composition of rose (Rosa canina L.) seed and oils. J. Med. Food, 5: 137-140.

POPEK R. 1996. Biosystematyczne studia nad rodzajem Rosa L. w Polsce i krajach ościennych. Wydawnictwo Naukowe WSP. Kraków.

RAJAPAKSE S., HUBBARD M., KELLY J.W., ABBOTT A.G., BALLARD R.E. 1992. Identification of rose cultivars by restriction fragment length polymorphism. Sci. Hortic. 52: 237-245 .

RAYMOND O., BIOLLEY J.P., JAY M. 1995. Fingerprinting the selection process of ancient roses by means of floral phenolic metabolism. Biochem. Systems Ecol. 23: 555-565.

RAZUNGLES A., OSZMIAŃSKI J., SAPIS J. 1989. Determination of carotenoids in fruits of Rosa ssp. (R. canina and $R$. rugosa) and of chokeberry (Aronia melanocarpa). J. Food Sci. 54: 774-775.

REIN E., KHARAZMI A., WINTHER K. 2004. A herbal remedy, Hyben Vital (stand. powder of a subspecies of Rosa canina fruits), reduces pain and improves general wellbeing in patients with osteoarthritis-a double-blind, placebo-controlled, randomized trial. Phytomedicine 11: 383-391.

SALAS J.J., SANCHEZ J., RAMLI U.S., MANAF A.M., WILLIAMS M., HARWOOD J.L. 2000. Biochemistry of lipid metabolism in olive and other oil fruits. Prog. Lipid Res. 39: 151$-180$.

SANINA N.M., GONCHAROVA S., KOSTESKY E.Y. 2004. Fatty acid composition of individual polar lipid classes from marine macrophytes. Phytochemistry 65: 721-730.

STEPANOV L., KADŽIJSKI T., PALAVEEVA T.S. 1983. Study of the composition of Rosa canina seeds. Maslo-Sap. Prom-st. 19: 38-44. 
STOŁYHWO A., MARTIN M., GUIOCHON G. 1987. Analysis of lipid classes by liquid chromatography with an evaporative light scattering detector. J. Liq. Chrom. 1237-1253.

SZENTMIHALYI K., VINKLER P., LAKATOS B., ILLES V., THEN M. 2002. Rose hip (Rosa canina L.) oil obtained from waste hip seeds by different extraction methods. Biosour. Technol. 82: 195-201.

UEDA Y., OKADA Y. 1994. Discrimination of rose cultivar groups by pollen surface structure. J. Hort. Sci. 69: 601-607.

UMEZU T., ITO H., NAGANO K., YAMAKOSHI M., OOUCHI H., SAKANIWA M., MORITA M. 2002. Anticonflict effects of rose oil and identification of its active constituents. Life Sci. 72: 91-102.

VALESCO L., GOFFMAN D. 1999. Chemotaxonomic significance of fatty acids and tocopherols in Boroginaceae. Phytochemistry 52: 423-426.
WINTHER K., REIN E., KHARAZAMI A. 1999. The anti-inflammatory properties of rose-hip. Inflammopharm. 7: 63.

YOKOI M. 1975. Colour and pigment distribution in the cultivars of selected ornamental plants, with special reference to their contribution to the ornamental value of plants. Trans. Faculty Hort. 14: 33-43.

ZIEGLER R.G. 1989. A review of epidemiologic evidence that carotenoids reduce the risk of cancer. J. Nutr. 119: 116-122.

ZIELIŃSKI J. 1985. Studia nad rodzajem Rosa L. - Systematyka sekcji Caninae DC. em. Christ. Arboretum Kórnickie 30: 3109. (in Polish)

ZIELIŃSKI J. 1987. Rosa L. in Flora Polski. PWN, Kraków. (in Polish)

ZLATANOV M.D. 1989. Lipid composition of Bulgarian chokeberry, black currant and rose hip seed oils. J. Sci. Food Agric. 79: $1620-1624$. 